\title{
Nutrición deportiva en la infancia: Cumplimiento de las demandas metabólicas del crecimiento y el ejercicio
}

\author{
C.L. Zanker \\ Facultad de Deporte y Educación Carnegie, Universidad Metropolitana de Leeds, Leeds, Reino Unido
}

\section{Palabras clave}

Ejercicio, nutrición · Crecimiento/pubertad, nutrición deportiva $\cdot$ Rendimiento físico $\cdot$ Metabolismo energético

\section{Extracto}

Los objetivos fundamentales de la infancia son el crecimiento y la maduración óptimos. Estos procesos relacionados con el desarrollo exigen un balance energético y nitrogenado positivo. Aunque la actividad física regular puede intensificar el desarrollo en la infancia, un entrenamiento físico extenuante puede reducir las reservas de energía y nitrógeno del organismo que, entonces, deberán reemplazarse a través de la alimentación. Los adolescentes físicamente activos son propensos a trastornos del comer, que pueden dar lugar a una malnutrición proteico-energética. Entre las consecuencias de un balance energético y nitrogenado negativos, destacan el retraso o la detención del crecimiento y la maduración puberal retardada. Actualmente las pruebas disponibles son insuficientes para asegurar que el entrenamiento físico ejerce un impacto negativo directo sobre el crecimiento y la maduración. Una alimentación bien equilibrada es esencial para el mantenimiento del rendimiento físico y la conservación del estado de salud en todos los individuos físicamente activos, independientemente de su edad. Los alimentos proporcionan los elementos químicos necesa- rios para la producción de energía por medio de la ejercitación de la musculatura esquelética, la reposición después del ejercicio de las reservas de energía y la regeneración del músculo dañado. Las necesidades alimentarias de los niños físicamente activos varían a medida que aumenta el tamaño corporal y progresa la pubertad. El estado de maduración es un determinante importante de las respuestas metabólicas y fisiológicas al ejercicio $y$, en consecuencia, influyen tanto sobre la capacidad del rendimiento físico como sobre las necesidades alimentarias. Estudios de metabolismo energético en niños dan a entender que, si bien están bien dotados para una actividad aerobia persistente, su capacidad para la actividad anaerobia está limitada debido a su estado de maduración. Es importante destacar que restricciones éticas impiden el tipo de investigación detallada e invasora que ha sido empleada para determinar las necesidades nutricionales en adultos físicamente activos. No obstante, parecería que, lo mismo que estos adultos, los niños físicamente activos presentan mayores demandas alimentarias con respecto a energía, hidratos de carbono, proteínas y agua que sus contrapartes sedentarias. En este artículo se justifican las necesidades nutricionales de niños atletas basándose en las demandas fisiológicas y metabólicas de supervivencia, crecimiento y actividad física.

Copyright ๑ 2006 Nestec Ltd., Vevey/S. Karger AG, Basel

\section{KARGER}

Fax +4161306 1234

E-Mail karger@karger.ch

www.karger.com (c) 2006 Nestec Ltd., Vevey/S. Karger AG, Basel

$0252-8185 / 06 / 0642-0063 \$ 23.50 / 0$

Accesible online en:

www.karger.com/ans
Dr. Cathy L. Zanker

Carnegie Research Institute

Carnegie Faculty of Sport and Education, Leeds Metropolitan University

Headingley Campus, Leeds LS6 3QS (UK)

Tel. +44 113283 2600, Fax +44 113283 7575, E-Mail c.zanker@leedsmet.ac.uk 


\section{Introducción}

La infancia representa una fase significativa de crecimiento y maduración física. Estos procesos dependen de una actividad física regular y una nutrición adecuada. También durante la infancia se desarrolla la personalidad y aparecen los talentos, entre los que destacan la aptitud atlética y la destreza en diversos deportes. Un entrenamiento físico cuidadosamente estructurado, en conjunción con una alimentación bien equilibrada, puede fomentar el rendimiento atlético en la infancia y preparar el cuerpo para futuras competiciones deportivas.

Antes de la pubertad, la capacidad de rendimiento físico de los niños y las niñas es bastante comparable. No obstante, a medida que progresa la pubertad empieza a manifestarse una divergencia significativa entre los sexos $[1,2]$. En ambos sexos, el entrenamiento físico aumenta los incrementos puberales normales del tamaño, la fuerza y la potencia aerobia y anaerobia de la musculatura, todos los cuales son determinantes importantes del rendimiento físico. No obstante, en las niñas, el desarrollo de estos rasgos fisiológicos y de rendimiento a través de la adolescencia es mucho menos prominente que en los niños; además, la acumulación significativa de grasa corporal en las muchachas puede impedir la aptitud atlética $[1,3]$. Un entrenamiento extenuante y un bajo peso corporal han sido asociados a disfunción neuroendocrina, supresión gonadal y múltiples trastornos endocrinos que trastornan el equilibrio normal del recambio óseo $[4,5]$. Entre las consecuencias de estos trastornos destacan el menoscabo del crecimiento, el retraso o la detención de la pubertad y la formación insuficiente de hueso, todo lo cual puede deteriorar la salud actual y futura $[1,3]$.

Cabe destacar, por su importancia, la existencia de pruebas convincentes de que los trastornos endocrinos y el metabolismo óseo desequilibrado en niños atletas son atribuibles en gran medida a una insuficiencia nutricional primaria, que es exacerbada en mayor medida por el coste energético añadido del entrenamiento físico que por el propio entrenamiento [6,7]. Basándose en esta prueba, es esencial que niños que participan en deportes extenuantes reciban una dieta que satisfaga adecuadamente las demandas nutricionales, tanto de su entrenamiento físico como de su crecimiento normal. En este artículo se justifican las necesidades nutricionales de niños atletas basándose en las demandas fisiológicas y metabólicas de supervivencia, crecimiento y actividad física. Se resumen los efectos del crecimiento y la maduración sobre las respuestas metabólicas y fisiológicas al ejercicio y se discute el impacto cooperativo de tales factores sobre el rendimiento físico y las necesidades alimentarias.

\section{Cuestiones éticas en relación con la investigación en niños atletas}

Al considerar las exigencias nutricionales de niños atletas, debe reconocerse que la base de las pruebas que orientan tales exigencias es limitada. Este hecho contrasta con las extensas pruebas de investigación que abordan las características nutricionales principales asociadas al ejercicio en adultos. Estas pruebas han sido utilizadas para elaborar normas alimentarias para hombres y mujeres que se entrenan para diversos deportes y compiten en los mismos [8]. La relativa falta de pruebas de investigación comparables en niños atléticos es atribuible principalmente a restricciones éticas y metodológicas sobre el tipo de recopilación de datos, restricciones que se han incrementado en el curso de los últimos años $[9,10]$. Las técnicas que se han aplicado en estudios metabólicos en adultos deportistas, aunque informativas, rebasan frecuentemente los límites de aceptabilidad ética para uso en niños.

En adultos, la medición de los combustibles transportados por la sangre e intramusculares o sus metabolitos ha propiciado el conocimiento de las tasas de recambio de sustratos energéticos durante el ejercicio. Además, el empleo de isótopos estables ha permitido investigar la cinética de la movilización y la utilización de sustratos. Estos procedimientos son sumamente informativos e instrumentales para el estudio detallado del balance energético y el metabolismo energético durante el ejercicio. No obstante, se acompañan de un grado de riesgo, malestar o dolor, que se considera excesivo o difícil de cuantificar en niños. En consecuencia, la investigación que implica procedimientos invasivos se autoriza, en general, sólo para uso en niños enfermos sometidos a tales procedimientos como parte de su asistencia sanitaria $[9,10]$. Al estudiar diversos aspectos del metabolismo en niños sanos, los preceptos éticos internacionales para la investigación biomédica en humanos [11] hacen hincapié en el empleo de técnicas indirectas que no son invasoras ni se entremeten en los hábitos de vida de un niño. Estos preceptos limitan claramente la intensidad y la precisión de la investigación que puede realizarse en niños, pero lo importante es que protegen la dignidad, la salud y el bienestar de los sujetos menores.

\section{Constitución de normas nutricionales para individuos físicamente activos}

Los determinantes principales de las necesidades nutricionales del ejercicio se refieren a las demandas de energía 'extra' de una actividad más allá de las demandas 
sedentarias y el consumo absoluto de diferentes combustibles metabólicos $[12,13]$. La hidratación ejerce también una influencia significativa sobre el rendimiento físico y la salud, y el consumo de líquidos a partir de alimentos y bebidas equilibraría las pérdidas de líquidos a través de la excreción y la transpiración. En la investigación en adultos se han abordado diversas cuestiones nutricionales relacionadas con el ejercicio, muchas de las cuales se resumen en la Declaración de Posición Conjunta sobre Nutrición y Rendimiento Atlético del Colegio Norteamericano de Medicina Deportiva (ACSM), la Asociación Dietética Norteamericana (ADA) y los Dietistas de Canadá (DC) [8]. Entre estas cuestiones nutricionales destacan las siguientes:

(1) Las demandas energéticas y los costes metabólicos específicos de diversos deportes y actividades [14, 15].

(2) La regulación del metabolismo energético en la ejercitación muscular, realizada con diferentes intensidades de ejercicio y durante períodos variables $[16,17]$.

(3) Diferencias de sexo en el metabolismo energético durante el ejercicio $[18,19]$.

(4) El papel de la manipulación de los alimentos, incluyendo la cronología del consumo de comidas en relación con el ejercicio y el uso de complementos alimentarios para modular el rendimiento físico y/o intensificar la recuperación después del ejercicio [20,21].

Las necesidades alimentarias establecidas para adultos físicamente activos se centran en la optimación del rendimiento y el mantenimiento de un buen estado de salud [8]. No obstante, gran parte de este conocimiento no existe en niños, y el grado en el cual los datos de los estudios en adultos son válidos, para los niños es actualmente dudoso. El interés nutricional fundamental para niños atletas es el suministro de energía suficiente y nutrientes específicos para el crecimiento y la maduración normales; el potencial ergógeno (es decir, intensificador del rendimiento) del alimento es de importancia secundaria [3, 22]. Por otra parte, la fisiología de los niños es menos adelantada que la de los adultos. Esto resulta especialmente cierto en niños prepuberales que, en comparación con los adultos, presentan respuestas desiguales al ejercicio en términos de gasto energético para una actividad dada, pautas de metabolismo energético y termorregulación [2, 22,23 . No obstante, se dispone de pruebas que dan a entender que las respuestas metabólicas y fisiológicas de adolescentes mayores, a diversas actividades, son comparables con las de adultos del mismo sexo [2, 22, 23].

El conocimiento actual de las necesidades alimentarias especiales de niños atletas de diferentes edades cronológicas o biológicas es bastante limitado. En consecuencia, las normas nutricionales para atletas infantiles son bastante ambiguas y se basan en los tres factores principales siguientes:

(1) Un conocimiento establecido de las demandas nutricionales del crecimiento y la maduración en la infancia.

(2) El potencial de rendimiento fisiológico y físico de niños de una edad cronológica o biológica dada.

(3) Adaptaciones de datos de adultos obtenidos de investigaciones metabólicas realizadas en asociación con ejercicio y entrenamiento físico.

En última instancia, los indicadores vitales de la idoneidad nutricional de la alimentación de un atleta infantil son el estado de salud y bienestar general del niño, su tasa de crecimiento y maduración lineales y lo apropiado de su ganancia de peso durante un período de tiempo específico $[1,7,22]$. En atletas infantiles, puede considerarse que la dieta satisface las demandas nutricionales de entrenamiento y crecimiento normal cuando, durante un período de meses, el niño sigue creciendo y aumentando de peso oportunamente, con los percentiles normales en una gráfica de crecimiento validada, y no existe retraso ni deterioro del desarrollo puberal $[1,7]$. La presencia de una carencia crónica de energía o proteína en niños viene indicada por incrementos insuficientes de la talla y el peso que se desvían de curvas de crecimiento validadas, o por una deficiencia del peso por altura, tal como se indica por medio de la evaluación del índice de masa corporal. Los cambios conductuales, incluyendo fatiga, letargia, irritabilidad y apatía, o la malfunción física, como infecciones frecuentes y lesiones en los tejidos blandos, pueden también significar insuficiencia nutricional, incluyendo deshidratación, en atletas infantiles $[22,24]$.

\section{Vías respiratorias en la ejercitación de la musculatura esquelética}

La energía para la actividad muscular se obtiene del catabolismo de la adenosina trifosfato (ATP). La cantidad de ATP presente en las células musculares es limitada y sólo suficiente para impulsar la actividad muscular durante unos pocos segundos. Para prolongar el ejercicio, la ATP debe ser, en consecuencia, sintetizada tan rápidamente como es utilizada $[12,13]$. En el curso del ejercicio, la actividad de las diversas vías respiratorias para la síntesis de ATP dentro de los músculos reclutados determina la velocidad y el grado del catabolismo de los combustibles almacenados y circulantes, influyendo de este modo sobre las demandas nutricionales del ejercicio $[12,13]$. En adultos y niños funcionan tres vías respiratorias principales dentro de la musculatura esquelética en funcionamiento: 
(1) La reacción de creatinfosfocinasa consiste en la fosforilación de la adenosina difosfato a través del catabolismo anaerobio de la creatina fosfato (PCr). Proporciona energía para descargas cortas ( $<10 \mathrm{~s})$ de actividad muscular de intensidad máxima, y es un sistema energético de vida corta debido a las cantidades limitadas de $\mathrm{PCr}$ depositadas.

(2) La glucolisis anaerobia implica el catabolismo anaerobio de glucógeno muscular a ácido láctico. Proporciona energía para un ejercicio breve $(<1 \mathrm{~min})$ de intensidad máxima y es un sistema energético de vida corta debido a que el producto secundario principal (ácido láctico), entre otros desechos metabólicos, interfiere con el acoplamiento excitación-contracción en la musculatura esquelética.

(3) La respiración aerobia representa el catabolismo completo de los combustibles (principalmente glucosa y ácidos grasos) a través del ciclo de los ácidos tricarboxílicos y la fosforilación oxidativa para formar bióxido de carbono y agua. Proporciona energía durante un período prolongado, dada la ausencia de acumulación de metabolitos tóxicos.

Tanto en adultos como en niños, el factor principal que determina las vías respiratorias operativas durante el ejercicio es la intensidad de la actividad muscular y, en consecuencia, la tasa de degradación de ATP $[12,16]$. La duración del ejercicio es también un factor influyente, dado que, a medida que la actividad muscular continúa, los depósitos de glucógeno intramusculares y hepáticos son vaciados selectivamente y los combustibles alternativos son reclamados en forma creciente $[17,20]$. Aparece fatiga cuando la fuerza de contracción muscular declina y un nivel dado de intensidad del ejercicio es insostenible. El agotamiento denota un punto en el cual deja de ser posible continuar el ejercicio [25]. La fatiga y el agotamiento están vinculados normalmente a la depleción del sustrato energético, a una acumulación de desechos metabólicos, a la deshidratación y/o a la hipertermia [25].

\section{Combustibles para la producción de energía}

Con arreglo al funcionamiento de las vías respiratorias mencionadas anteriormente, los combustibles principales para la síntesis de ATP derivados de la alimentación, dentro de los músculos en funcionamiento, tanto en adultos como en niños, son los depósitos en los propios músculos de $\mathrm{PCr}$, glucógeno y triglicéridos, conjuntamente con la glucosa y los ácidos grasos no esterificados transportados por la sangre $[16,17]$. Durante el ejercicio prolongado, las fuentes energéticas que limitan al orga- nismo son el glucógeno y la glucosa derivados de los hidratos de carbono alimentarios $[17,20]$. La velocidad y el grado del catabolismo de estos combustibles están regidos por la intensidad y la duración del ejercicio [26, 27]. A pesar de ser tan ricos en energía, como la glucosa por masa unitaria, los aminoácidos contribuyen sólo en proporción menor a la síntesis de ATP durante el ejercicio en sujetos sanos, bien nutridos, excepto en casos de ejercicio prolongado (por ejemplo, >90 min) e intenso (por ejemplo, $>75 \%$ de potencia aerobia máxima o $\dot{\mathrm{V}}_{2 \text { máx }}$ ) [17, 20].

El ejercicio prolongado y extenuante puede vaciar en última instancia las reservas de glucógeno musculares y hepáticas, dado que la ATP debe ser sintetizada a un ritmo elevado durante un período prolongado $[20,27]$. El glucógeno muscular y la glucosa sanguínea contribuyen significativamente a dicha operación. Dado que el glucógeno se almacena precariamente con el ejercicio continuado, con aparición de hipoglucemia, un descenso del cociente entre las concentraciones plasmáticas de insulina y glucagón y un incremento de la concentración plasmática de cortisol estimulan la proteolisis muscular [21, 28]. Dicha proteolisis da lugar a la liberación de aminoácidos libres. Los músculos activos son capaces de extraer y oxidar cantidades crecientes de los aminoácidos de cadena ramificada, leucina, isoleucina y valina, mientras que otros aminoácidos pueden ser transaminados para producir alanina y glutamina destinadas a la gluconeogénesis hepática $[28,29]$. No obstante, los aminoácidos no son los combustibles 'ideales' para la síntesis de ATP, dado que el catabolismo intensificado de las proteínas tisulares plantea el riesgo de un balance nitrogenado negativo. Cuando éste es persistente, se deteriora la salud y puede aparecer inmunosupresión, lesiones en los tejidos blandos y deterioro de la formación ósea [4, 21]. Debido a la importancia de un balance nitrogenado positivo para el crecimiento, es probable que las consecuencias nocivas para la salud de la depleción de glucógeno inducida por el esfuerzo sean mayores en niños que en adultos.

La movilización de los lípidos y la oxidación de los ácidos grasos también aumentan con la duración del ejercicio, tanto en niños como en adultos $[26,30]$. Este incremento del metabolismo de las grasas es inducido por múltiples respuestas endocrinas, entre las que destacan incrementos de los niveles plasmáticos de cortisol, catecolaminas y hormona del crecimiento y disminución de la concentración plasmática de insulina [31,32]. Es interesante destacar que estudios basados en la calorimetría indirecta para evaluar el cociente de intercambio respiratorio no proteínico $\left(\mathrm{V}_{2} / \mathrm{V} \mathrm{CO}_{2}\right)$ indican que la contribución de la oxidación de los ácidos grasos a la producción 
de energía es superior en niños que en adultos que se ejercitan con una misma intensidad de ejercicio relativa (expresándose esta última como $\% \mathrm{~V}_{2}{ }_{2 \text { máx }}$ ) [33-35]. Esta tendencia parece menguar durante la adolescencia, especialmente en varones $[23,36]$. Esto indica que las hormonas sexuales, especialmente los andrógenos, desempeñan un papel en la regulación del metabolismo de los combustibles. Entre las implicaciones nutricionales de mayor capacidad para oxidar ácidos grasos en los niños prepuberales, destacan la conservación del glucógeno y la glucosa en la sangre, que puede reducir las necesidades de hidratos de carbono alimentarios.

\section{Determinantes físicos y fisiológicos del rendimiento físico}

La excelencia en el rendimiento deportivo depende de características físicas y fisiológicas hereditarias ('genes buenos'), un entrenamiento físico bien estructurado para fomentar características innatas y una nutrición sólida para facilitar la adaptación óptima al entrenamiento [37]. Los principales determinantes físicos y fisiológicos del rendimiento deportivo influidos por la nutrición son la fuerza, la velocidad, la potencia y la resistencia. La importancia relativa de estas características para el rendimiento deportivo varía de un deporte a otro y es insólito que coincidan todos los atributos cruciales para la destreza en un deporte dado. Estas características físicas y fisiológicas se definen a continuación [37].

El término fuerza denota la capacidad para generar fuerza, y está regida por el tamaño de los músculos individuales y los músculos agonistas, en conexión con la coordinación de las unidades motoras de estos músculos. La fuerza es una determinante clave de la potencia, que es igual al producto de la fuerza muscular por la velocidad de contracción. Entre los deportes que exigen fuerza y potencia extremas destacan correr a toda velocidad (carreras, natación o ciclismo), saltar, lanzar y levantar pesas. La gimnasia, la lucha, los deportes de raqueta y numerosos deportes en equipo incorporan también maniobras de potencia. La excelencia del rendimiento en la mayoría de los deportes depende de un cociente elevado entre la fuerza (o la potencia) y la masa corporal. Esto atañe especialmente a deportes que implican movimiento del cuerpo frente a la fuerza debida a la gravedad (por ejemplo, correr, saltar, practicar gimnasia, etc.). En contraste, en deportes como la natación y el ciclismo, donde la masa corporal está sustentada, la fuerza y la potencia absolutas son de mayor importancia para el rendimiento deportivo. La actividad muscular durante la realización

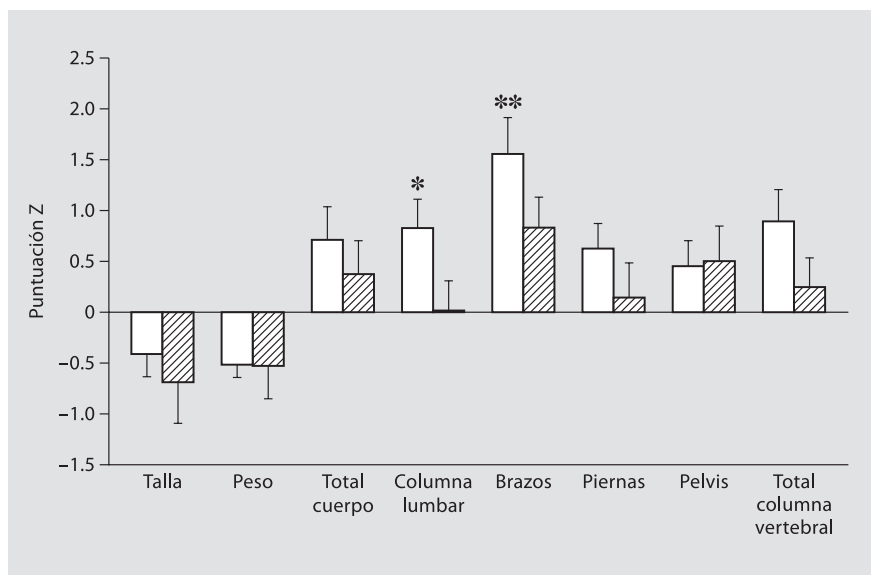

Fig. 1. Talla, peso y densidad mineral ósea (DMO) de zonas regionales de gimnastas infantiles masculinos y femeninos (de 6 a 8 años de edad), expresados en puntuaciones estandarizadas $(Z)$ en relación con los valores medios de niños no entrenados (controles) del mismo sexo y talla. $\square$ = Niñas; $\square=$ niños. Aunque ambos grupos de gimnastas eran pequeños y livianos para su edad, mostraban tendencias a favor de una mayor DMO que los controles. Las gimnastas femeninas se entrenaban de 8 a $10 \mathrm{~h}$ por semana, $\mathrm{y}$ así habían procedido con regularidad durante 3 a 4 años. Los gimnastas masculinos entrenaban 4 a $6 \mathrm{~h}$ por semana, y su entrenamiento se remontaba a un período de 1 a 2 años. Significación, gimnastas frente a controles del mismo sexo: ${ }^{*} \mathrm{p}<0,05 ;{ }^{* *} \mathrm{p}<$ 0,01. De Zanker et al. [39].

de estos deportes está sustentada fundamentalmente por la energía generada a través del catabolismo anaerobio de depósitos musculares de creatina fosfato y glucógeno. Estos sistemas respiratorios anaerobios permiten una tasa de síntesis de ATP considerablemente superior a la que pueda ser generada a través de la respiración aerobia.

El término resistencia describe la capacidad para seguir ejercitándose sin fatiga y viene determinada en gran medida por la buena salud cardiorrespiratoria, conjuntamente con la capacidad del músculo en funcionamiento para extraer y utilizar oxígeno en la respiración aerobia. Como ejemplos de deportes de resistencia mencionaremos las carreras, la natación y el ciclismo a largas distancias, el remo y el esquí campo traviesa. Aparte de la exigencia de fuerza y potencia, los deportes de raqueta y en equipo demandan también cierto grado de resistencia cardiorrespiratoria y muscular. Los deportes de resistencia, conjuntamente con su entrenamiento, implican el coste energético más elevado de todas las actividades físicas. Aunque la tasa de gasto de energía es menor en los deportes de resistencia que en los deportes de fuerza y potencia, la cantidad total de energía consumida durante la actividad de resistencia es superior debido a la mayor duración para la cual está sustentada. Las actividades de 
resistencia se basan predominantemente en la respiración aerobia, que utiliza diversas fuentes de combustible metabólico, en función de la intensidad y la duración del ejercicio, conjuntamente con la magnitud de los depósitos de glucógeno de los músculos activos.

\section{Desarrollo de la destreza atlética con el crecimiento y la maduración}

En el momento de explorar las necesidades alimentarias de los atletas infantiles, es necesario considerar los factores que influyen sobre las necesidades de energía y nutrientes específicos individuales de un niño. En los niños en fase de crecimiento, entre estos factores destacan el estado de salud general, el tamaño físico, las dimensiones corporales, el estado de maduración y la actividad física $[1,22,23]$. Esta última engendra demandas nutricionales específicas, que guardan relación con la provisión de combustible para la actividad muscular y la promoción de un entorno anabólico para facilitar la recuperación después del ejercicio. El tamaño corporal y el estado de maduración son determinantes fundamentales de la capacidad del rendimiento físico en niños y, en el curso del desarrollo infantil, particularmente durante la pubertad, los incrementos de la fuerza muscular y la potencia aerobia fomentan la destreza atlética $[1,22,23]$.

En los niños más pequeños ( $<8$ años), la actividad física espontánea produce mayores incrementos del rendimiento físico en relación con el crecimiento. Las carreras, los saltos y las maniobras acrobáticas son características naturales del juego infantil, que mejoran la coordinación, las destrezas motoras, la fuerza muscular y la potencia aerobia [38]. Por otra parte, en presencia de una nutrición adecuada, estas actividades parecen intensificar la adquisición prepuberal de masa muscular y ósea [39]. En la figura 1 se ilustran diferencias en la densidad ósea entre gimnastas infantiles y niños normalmente activos de 7 a 8 años de edad, y se demuestran los efectos manifiestamente positivos de las actividades de soporte de peso y gran impacto sobre la acreción regional de mineral óseo. Durante esta fase de desarrollo inicial, el entrenamiento intensivo es excepcional y, en general, desatinado debido a la propensión del sistema músculo esquelético en crecimiento a los traumatismos por uso excesivo [2]. No obstante, para actividades deportivas como la gimnasia y el ballet, el entrenamiento controlado es habitual y esencial para el fomento y el mantenimiento de la flexibilidad, que en caso contrario se deterioraría con el crecimiento $[2,40]$.

A medida que se incrementa la edad y la madurez, los niños son capaces de resistir un entrenamiento físico más duro y estructurado, especialmente tras el inicio de la pubertad. Antes de ésta, independientemente de la nutrición, la musculatura esquelética no es capaz de hipertrofiarse significativamente, aunque son posibles incrementos de la fuerza de mediación neural $[1,23]$. Hasta el inicio de la pubertad no es posible que ondas de impulso de los niveles circulantes de hormonas sexuales, hormona del crecimiento y factores de crecimiento de tipo insulínico permitan respuestas hipertróficas de la musculatura esquelética a actividades que requieran fuerza y potencia. La fuerza muscular por área transversal unitaria es comparable en niños, adolescentes y adultos jóvenes de ambos sexos; el entorno endocrino en diferentes etapas de madurez en niños y niñas determina la capacidad para incrementos de la masa muscular inducidos por el ejercicio $[1,23]$. Las hormonas sexuales, particularmente los andrógenos, pueden propiciar también la glucolisis anaerobia en la musculatura esquelética, dado que la capacidad anaerobia y la producción de lactato son considerablemente superiores en hombres que en niños prepuberales, niñas o mujeres $[23,36]$. Se ha dado a entender que los andrógenos inducen las enzimas que controlan la glucolisis anaerobia e incrementan la capacidad de almacenamiento de glucógeno $[23,36]$.

Aunque $\mathrm{V}_{2 \text { máx }}$ es independiente del estado de maduración cuando se expresa en relación con la masa corporal, es superior en individuos de mayor tamaño (por ejemplo, en adultos que en niños) cuando se expresa en términos absolutos [23, 41]. Esto se debe a que un determinante principal de $\mathrm{V}_{2 \text { máx }}$ es el gasto cardiaco máximo, mientras que el volumen sistólico máximo está relacionado con el tamaño del corazón en sujetos sanos. $\dot{V}_{2 \text { máx }}$ depende también de la capacidad de una gran masa de músculo en funcionamiento para extraer el oxígeno transportado por la sangre y utilizarlo en la respiración aerobia. La función pulmonar adecuada y la concentración de hemoglobina en la sangre influyen inevitablemente sobre la capacidad de transporte de oxígeno [42, 43]. En comparación con adultos y adolescentes mayores, los niños más pequeños muestran una eficiencia mecánica y una economía de utilización de oxígeno menores al caminar y al correr [2,22]. Cabe mencionar el ejemplo de las mediciones del consumo submáximo de oxígeno $\left(\mathrm{V}_{2}\right)$, expresado por masa corporal unitaria, que es superior en niños que en adultos que realizan una actividad dada.

Se ha dado a entender que una menor economía de utilización de oxígeno en los niños más pequeños guarda relación con una coordinación menos eficaz de los grupos musculares agonistas y antagonistas, con el consiguiente 'desperdicio' de energía [2, 22]. Dado que $\mathrm{V}_{2}$ es proporcional al gasto de energía durante el ejercicio submáximo 
en estado de equilibrio, las tablas que relacionan las demandas energéticas de diversas actividades físicas, compiladas a partir de la investigación en adultos [14], suelen subestimar los gastos de energía de niños que emprenden las mismas actividades, incluso cuando se convierte a escala para tener en cuenta las diferencias en el tamaño y la masa corporales. Se ha estimado que en niños prepuberales y en adolescentes, el gasto de energía para una actividad dada excede de los valores en adultos en el 20 al 25\% y el 10 al $15 \%$, respectivamente $[2,22]$.

La conclusión principal que puede extraerse con respecto a los efectos del entrenamiento físico en niños prepuberales es que dicho entrenamiento específico puede intensificar el desarrollo de las habilidades motoras, la fuerza muscular y la potencia aerobia. No obstante, en contraste con adolescentes y adultos, el potencial de los niños más pequeños para incrementar la potencia aerobia y anaerobia, a través del entrenamiento específico, es comparativamente limitado $[2,22]$. La modulación de la ingesta alimentaria (por ejemplo, una elevación del consumo de proteínas más allá de lo exigido para el mantenimiento del balance nitrogenado) no puede incrementar estas características de rendimiento antes de la pubertad [22, 24]. Durante la pubertad, los incrementos de la fuerza del cuerpo entero, la potencia muscular y la resistencia presentan una magnitud considerablemente superior en niños que en niñas, tanto cuando se expresan en términos absolutos como en relación con la masa corporal $[2,22]$. Estas diferencias entre los sexos se explican por diferencias en el tamaño corporal, la composición corporal y el metabolismo entre adolescentes y adultos. Mientras que los andrógenos (especialmente la testosterona) en varones adolescentes estimulan el crecimiento del cuerpo entero e incrementan la masa tisular magra, los estrógenos en las adolescentes aumentan la reposición de grasa corporal y el cociente entre tejido graso y tejido magro. Por otra parte, los efectos anabólicos de los estrógenos son significativamente menores que los de los andrógenos [2, 22]. Las diferencias entre sexos en el grado de desarrollo de las características del rendimiento físico durante la adolescencia explican las diferencias confirmadas en el potencial del rendimiento deportivo de hombres y mujeres adultos $[2,43]$.

\section{Papel de los alimentos y los factores que influyen sobre las necesidades nutricionales básicas de adultos y niños}

El alimento proporciona el sustrato energético imperativo para el metabolismo y el mantenimiento de la homeostasis. El balance energético, que representa la dife- rencia entre la ingesta y el gasto de energía, es fundamental para el estado de salud y el rendimiento físico. En adultos, las necesidades de energía alimentaria reflejan los costes energéticos del metabolismo basal, el efecto térmico de los alimentos, la termorregulación y la actividad física $[8,13]$. Esta última comprende las actividades rutinarias de la vida cotidiana, así como el deporte y el ejercicio estructurado. La actividad física produce un efecto intenso sobre la tasa de gasto de energía, y el ejercicio que implica el reclutamiento de una considerable masa muscular eleva en gran medida el ritmo metabólico en reposo (RMR). Por ejemplo, en adultos durante la actividad aerobia persistente en el cuerpo entero ( $>5 \mathrm{~min}$.), el RMR puede aumentar de 10 a 20 veces, mientras que durante la actividad anaerobia de intensidad máxima y duración corta ( $<1 \mathrm{~min}$.), cabe la posibilidad de una elevación momentánea del RMR de 40 a 50 veces [13]. En consecuencia, la repetición de ejercicios extenuantes al servicio de un entrenamiento físico conlleva el potencial de inducir una deficiencia energética a menos que ésta sea contrarrestada por una ingesa de energía alimentaria adecuada.

En niños, la energía es necesaria para las mismas funciones que en adultos; no obstante, se exige una cantidad extra más allá de las demandas del RMR para sustentar el crecimiento y la maduración. El crecimiento depende de la síntesis y la deposición netas de tejidos y, en consecuencia, es un proceso anabólico que incorpora la energía química procedente de los alimentos a los componentes estructurales de los órganos. Dado que la tasa de recambio tisular es considerablemente superior en niños que en adultos, el gasto de energía por masa corporal unitaria es también superior en los niños [22,44]. Por otra parte, para sustentar el crecimiento y la maduración normales, los niños deben mantener un balance nitrogenado positivo. Esto último requiere el consumo de nitrógeno (a partir de alimentos proteínicos) que exceda de la utilización de nitrógeno (en la síntesis y la producción de energía). Un balance energético negativo causa inevitablemente un balance nitrogenado negativo, dada la catabolización de las proteínas para generar aminoácidos destinados al uso como combustible metabólico [21, 45]. La consecuencia de dicho catabolismo, tanto en adultos como en niños, es una pérdida de peso que afecta tanto al tejido magro como al tejido adiposo. No obstante, en niños con deficiencia energética y balance nitrogenado negativo persistentes, puede producirse una desaceleración colateral del crecimiento, cuyo resultado es una talla baja para la edad cronológica. Este tipo de 'enanismo nutricional' se caracteriza por una declinación de la tasa de crecimiento lineal con ganancia de peso insuficiente y un retraso o incluso una detención de la pubertad $[7,40]$. 
La insuficiencia nutricional durante la etapa adolescente, especialmente de energía y proteína, genera un entorno catabólico que impide los acontecimientos anabólicos necesarios para el crecimiento y la maduración normales. Durante el brote de crecimiento puberal, las demandas de energía y proteína alimentarias alcanzan un máximo para garantizar el balance nitrogenado positivo $[22,24]$. A lo largo de toda esta fase de desarrollo, particularmente en muchachas adolescentes, el hipotálamo es especialmente sensible a la deficiencia energética aguda o crónica, la hipoglucemia y la reducción de los depósitos de grasa. Estas agresiones nutricionales perturban las variaciones diurnas normales en los niveles circulantes de insulina, 3,5,3'-triyodotironina, cortisol y leptina $[4,46]$. Se cree que esta última informa al hipotálamo de la disponibilidad de energía y regula indirectamente la función hipofisaria junto a las actividades de las glándulas endocrinas periféricas $[4,47]$. Los efectos nocivos de la carencia de energía y proteína sobre el crecimiento y el desarrollo puberal son atribuibles a disfunción neuroendocrina, que conduce a un trastorno generalizado de la síntesis y el metabolismo de las hormonas con consecuencias de largo alcance $[4,7]$. La supresión resultante de la función gonadal, asociada a alteraciones en el metabolismo o en las acciones periféricas de la hormona tiroidea y la hormona del crecimiento son particularmente perjudiciales para la integridad del esqueleto. En niños sanos, del 30 al $40 \%$ de la masa ósea del adulto se acumula normalmente durante la pubertad bajo la influencia anabólica de hormonas sexuales, factores de crecimiento de tipo insulínico y leptina [48, 49]. Una deficiencia de estas hormonas en condiciones de carencia de energía deteriora la formación ósea. El resultado es una masa ósea exigua y una estructura ósea frágil en la edad adulta $[4,49]$.

Mediante la rehabilitación nutricional, suele ser posible que niños previamente subnutridos incrementen su peso para experimentar un crecimiento 'adquirido' y completar la maduración puberal $[7,40]$. No obstante, si la insuficiencia nutricional persiste durante varios años, el crecimiento óseo y la adquisición mineral normales pueden quedar permanentemente deteriorados y no alcanzar jamás la talla adulta genéticamente 'programada'. En tales circunstancias, la maduración puberal es incompleta y se menoscaba la capacidad reproductora $[7,40]$. Si la insuficiencia nutricional aparece en torno a la edad de la pubertad prevista, el brote de crecimiento puberal y la subsiguiente maduración se retrasan o se inhiben $[7,40]$. Cuanto mayor sea la intensidad de la carencia energética en la infancia y más tiempo se soporte esta carencia, tanto más ominoso será el pronóstico de la consecución esperada del crecimiento y la maduración normales. En consecuencia, cuando se consideran las necesidades nutricionales de los atletas infantiles, es importante apreciar que sus dietas deben abarcar las demandas nutricionales específicas del crecimiento y la maduración, complementariamente a las demandas energéticas del entrenamiento físico.

No obstante, es digno de mención que algunos niños que destacan en ciertos deportes (por ejemplo, gimnasia y danza) son capaces de conseguirlo debido a una talla baja y una pubertad tardía hereditarias. Estos niños tienen a menudo padres y hermanos de baja estatura y presentan un retraso constitucional del crecimiento (es decir, un retraso inherente en el tiempo del crecimiento lineal y la ganancia de peso); no obstante, pueden estar adecuadamente nutridos $y$, por otra parte, estar sanos [7, 40]. Actualmente, se dispone de pruebas limitadas que permitan suponer que el retraso del crecimiento y la maduración observado en diversas cohortes de atletas infantiles sea directamente atribuible al entrenamiento físico de por sí. Por el contrario, si las demandas de energía y nutrientes específicos del entrenamiento están equilibradas a través de una nutrición adecuada, el crecimiento y la maduración progresan normalmente dentro de límites normales $[3,50]$.

\section{Trastornos del comer en atletas infantiles}

La insuficiencia nutricional primaria en niños físicamente activos en sociedades desarrolladas se debe habitualmente a una restricción alimentaria deliberada y voluntaria en lugar de a un acceso limitado a los alimentos. El término 'trastornos del comer' describe una pauta de comportamiento heterodoxo en relación con las comidas, que puede satisfacer a un número mayor o menor de criterios diagnósticos formales de un trastorno del comer clínico, como la anorexia o la bulimia nerviosa $[51,52]$. Aunque el origen de los trastornos del comer es normalmente psicógeno, este comportamiento puede perpetuarse por la malnutrición y las adaptaciones metabólicas colaterales que ejercen un impacto nocivo sobre la función cognitiva. Los trastornos clínicos del comer son más corrientes en niñas que en niños y aparecen habitualmente tras el inicio de la pubertad, cuando una onda de impulso de los niveles circulantes de hormonas sexuales produce cambios rápidos en la forma corporal, el cociente entre masa grasa y masa magra y estabilidad emocional errática. Los trastornos clínicos del comer se caracterizan por comportamientos rituales del comer, que se asocian frecuentemente a otros rasgos obsesivos y compulsivos, como la dependencia al ejercicio [51, 52]. 
Tabla 1. Factores principales que influyen sobre el gasto de energía y el metabolismo de los combustibles durante el ejercicio

Intensidad y duración del ejercicio, que influyen sobre el grado en el cual los sistemas energéticos aerobios y/o anaerobios de los músculos son requeridos y, en consecuencia, la 'elección' del combustible metabólico $[26,27]$

Frecuencia del entrenamiento, que influye sobre la capacidad para recuperar los depósitos de combustibles entre tandas repetidas de ejercicio $[17,20]$

Magnitud de los depósitos de combustibles dentro de las fibras musculares reclutadas, antes y después del ejercicio, que comprenden principalmente glucógeno y triglicéridos $[17,20]$

Concentración de glucosa en la sangre, que influye sobre la liberación de hormonas que mantienen el control glucémico, como la insulina, el cortisol, las catecolaminas y la hormona del crecimiento [31, 32]

Alimentación habitual, particularmente la ingestión de macronutrientes, que también influye sobre la magnitud de los depósitos de combustibles de los músculos y la concentración de glucosa en la sangre $[17,20]$

Tipo de fibra muscular, basado en propiedades estructurales y bioquímicas, incluyendo la actividad de enzimas que controlan el catabolismo y el almacenamiento de combustibles metabólicos $[12,17]$

'Estado' del entrenamiento, definido por el grado de adaptación metabólica al ejercicio específico, repetido, particularmente dentro de los músculos esqueléticos reclutados $[12,17]$

Niveles circulantes de hormonas sexuales, que son desdeñables en niños prepuberales y aumentan con el inicio de la pubertad $[23,36]$

El comer restrictivo y el ejercicio 'excesivo', pero fútil, son particularmente nocivos para el crecimiento, la maduración y la salud futura de los adolescentes. Se dispone de trabajos en los que se describe que los trastornos del comer restrictivo están más difundidos en determinadas cohortes atléticas (por ejemplo, corredores de distancia, gimnastas, bailarines y luchadores), que en individuos normalmente activos [51, 52]. Este dato podría explicarse por la presión hallada o percibida por estos jóvenes atletas para alcanzar y mantener un físico delgado o para mejorar el rendimiento o los atributos estéticos. No obstante, es también posible que los adolescentes con personalidad obsesiva o, por otra parte, vulnerable, se 'autoseleccionen' para tales deportes. Para estos adolescentes, un entrenamiento físico estructurado puede satisfacer una necesidad de rutina y ritual, y los clubs deportivos proporcionan un entorno en el cual la delgadez y la repetición de los ejercicios son esperadas y aceptadas $[51,52]$.

La insuficiencia nutricional en atletas en fase de crecimiento puede derivar también de una simple falta de conocimiento o una interpretación errónea de la importan-

Las demandas nutricionales del ejercicio cia de la alimentación para el mantenimiento del rendimiento y la salud, por parte del atleta o sus padres o preparadores. La confusión y la incertidumbre que rodean a las necesidades alimentarias del atleta infantil son comprensibles en función de la escasez de la investigación publicada sobre esta cuestión. Los niños, especialmente los adolescentes, pueden adoptar actitudes alimentarias peligrosas debido a que están sometidos a un asesoramiento alimentario engañoso o no confirmado, a través de los medios y las revistas populares. Por estos motivos, los atletas infantiles deben ser aconsejados cuidadosamente por personas poseedoras de la experiencia dietética apropiada. Los adolescentes tienden a ser excepcionalmente sensibles a la imagen corporal y pueden tener puntos de vista distorsionados de los alimentos y del comer. En lugar de comprender el comer como una actitud esencial para impulsar un metabolismo normal, pueden ver en los alimentos a un 'enemigo' que les fomentaría un aumento de peso indeseable $[24,51,52]$. En consecuencia, los niños atletas deben ser educados por otras personas pertinentes (por ejemplo, preparadores y padres) con respecto a la importancia de los alimentos como combustible vital para un rendimiento y un estado de salud óptimos.

\section{Nutrición deportiva general: la importancia especial de la nutrición para los atletas}

Una alimentación bien equilibrada es esencial para el mantenimiento del rendimiento físico y la conservación del estado de salud en todos los individuos comprometidos con un entrenamiento físico, independientemente de su edad. Los alimentos proporcionan los elementos químicos necesarios para la producción de energía por activación de la musculatura esquelética, reposición de los depósitos de combustibles después del ejercicio y regeneración del músculo dañado. La nutrición también modula los niveles circulantes de hormonas anabólicas y catabólicas, que influyen sobre el metabolismo de los combustibles almacenados durante el ejercicio y la capacidad para la recuperación después del mismo [21, 53]. El interés nutricional fundamental para individuos con un gasto elevado de energía durante el ejercicio es asegurar una ingesta de energía adecuada. Estos individuos presentan necesidades de energía y nutrientes específicos que exceden de las de sus contrapartes sedentarias de edad y sexo equiparables [8]. Estas necesidades están regidas por el grado en el cual el ejercicio genera un gasto energético por encima del RMR, la duración del gasto de energía elevado, el coste energético 'extra' total del ejercicio regular y el consumo y la posible depleción asociados de combus- 
Tabla 2. Funciones clave de la nutrición para la promoción de adaptaciones favorables al entrenamiento físico

Provisión de combustible metabólico a la musculatura esquelética durante el ejercicio para equilibrar la producción y la utilización de energía y, en consecuencia, prevenir la fatiga $[13,16]$

Reposición de los depósitos de combustibles después del ejercicio, especialmente glucógeno muscular y hepático, que son almacenados en cantidades limitadas en el organismo en comparación con la grasa $[13,16]$

Restablecimiento de la homeostasis tras el ejercicio, que incluye el reemplazo de líquidos y electrolitos, así como la normalización de la concentración de glucosa en la sangre $[21,53]$

Regeneración y reparación de los tejidos del sistema musculoesquelético, dañados por los microtraumatismos inducidos por el ejercicio, para fomentar una 'supercompensación' (es decir, una respuesta anabólica superior) $[21,53]$

tibles específicos. La alimentación, el balance energético y el entrenamiento físico también regulan la masa y la composición corporales, que influyen sobre la capacidad del rendimiento deportivo $[8,37]$.

También es probable que los niños y los adultos comprometidos con un entrenamiento físico regular se beneficien de un mayor consumo de macronutrientes y micronutrientes específicos, por encima de las recomendaciones para necesidades sedentarias. Se trata de contribuir a la producción de energía para el ejercicio y la regeneración tisular después del mismo. En la tabla 1 se presentan los factores principales que influyen sobre el gasto de energía y el metabolismo de los combustibles durante el ejercicio.

Una mayor intensidad del ejercicio y un mayor gasto energético se acompañan también de una mayor descomposición y utilización del glucógeno, en relación con la grasa, como combustible muscular, tanto en adultos como en niños $[26,44]$. Un ejercicio prolongado, de gran intensidad, en ausencia de ingestión de hidratos de carbono causa una reducción considerable de los depósitos de glucógeno musculares y hepáticos [16, 17]. Por lo tanto, para evitar la depleción de glucógeno y la fatiga, todos los individuos sometidos con regularidad a este tipo de ejercicio precisan una dieta energética equilibrada, de la cual por lo menos un 55\% de la energía derive de los hidratos de carbono $[8,24]$. La repetición de ejercicios que comprometan los mismos grupos musculares incrementa el riesgo de depleción de glucógeno dentro de las fibras musculares específicamente reclutadas. En consecuencia, las demandas nutricionales del entrenamiento físico están fuertemente vinculadas a la disponibilidad de hidratos de carbono, en forma de glucosa en la sangre y glucógeno en el hígado y el músculo [16, 17]. Debido a la abundancia de depósitos de grasa corporal, en relación con el glucógeno, en adultos y niños sanos, la disponibilidad de lípidos no limita normalmente el rendimiento del ejercicio. Por lo tanto, una dieta rica en grasas posee una eficacia limitada con miras a la actividad muscular y el rendimiento físico, la recuperación después del ejercicio y la capacidad de entrenamiento en individuos físicamente activos [20-22].

Las diferencias en el metabolismo de los combustibles, durante el ejercicio, entre adultos y niños son atribuibles principalmente a diferencias en el tamaño físico y los niveles contrastantes de hormonas sexuales circulantes [22, 23]. Estas hormonas influyen sobre el metabolismo de los combustibles a través de sus efectos sobre el tamaño y la composición corporales, la magnitud de los depósitos de combustibles intramusculares y extramusculares del organismo y la actividad de diversas enzimas que controlan las vías respiratorias dentro de la musculatura esquelética. Las discrepancias en los niveles circulantes de las diversas hormonas sexuales pueden también apuntalar variaciones en el metabolismo de los combustibles, observadas entre hombres y mujeres durante ejercicios de intensidad relativa dada $[18,19]$.

\section{Recuperación después del ejercicio}

El entrenamiento físico proporciona el estímulo esencial para los cambios adaptativos en la fisiología y el metabolismo, que pueden beneficiar o impedir el rendimiento y el estado de salud $[53,54]$. La nutrición, el balance energético y el nivel circulante de combustibles metabólicos desempeñan un papel primordial en el proceso de recuperación después del ejercicio. Este último describe la recuperación de la homeostasis tras el ejercicio e implica la repleción de depósitos de energía junto a la síntesis de proteínas estructurales y enzimas [53, 54]. La eficacia de la recuperación tras el ejercicio determina la naturaleza de las adaptaciones metabólicas y funcionales a una tanda de ejercicios subsiguiente. En particular, un ejercicio extenuante prolongado impone un estrés considerable sobre los sistemas de órganos principales e induce un catabolismo generalizado. Tras el ejercicio, este catabolismo es invertido a través de la combinación de reposo y nutrición sólida, que permiten un anabolismo neto. El anabolismo fomenta adaptaciones positivas en la función de los tejidos agredidos por el ejercicio [53, 54]. Una característica importante del reposo en el programa de entrenamiento reside en que se acompaña de una pauta de metabolismo de los combustibles en la musculatura esquelética que contrasta con la pauta que aparece duran- 
te el ejercicio [26, 53]. En lugar de catabolizar grandes cantidades de glucógeno, el sustrato energético predominante para la musculatura esquelética en reposo es la grasa. Las tasas bajas de oxidación de glucosa y glucógeno fomentan la resíntesis del glucógeno y, en consecuencia, la recuperación de las reservas de glucógeno $[26,53]$.

La alimentación desempeña un papel importante en las adaptaciones al entrenamiento físico. La incapacidad para sustituir los depósitos de combustibles después del ejercicio, especialmente el glucógeno muscular y hepático, impide una inversión del catabolismo inducido por el ejercicio, limita la capacidad para el ejercicio ulterior y puede inducir respuestas maladaptativas desfavorables $[21,54]$. La maladaptación prolongada o repetida puede dar también lugar a síntomas de 'sobreentrenamiento'. Este último se caracteriza por trastornos de la fisiología, el metabolismo y el estado de ánimo normales, que deterioran el rendimiento, el estado de salud y el bienestar [54, 55]. Aunque la mayor parte de la investigación sobre la interacción de dieta y entrenamiento físico sobre el rendimiento y el estado de salud ha sido realizada en adultos en lugar de en niños, dadas las necesidades especiales de nutrición óptima para el crecimiento en la infancia es lógico especular que la propensión de un niño al deterioro de la salud, cuando se intentan equilibrar las demandas que competen al crecimiento y al entrenamiento físico, es superior a la del adulto. Por lo tanto, la nutrición es un factor decisivo en la capacidad para aprovechar el entrenamiento físico, debido a sus efectos reguladores sobre el balance anabólico/catabólico prevaleciente.

En la tabla 2 se recopilan las funciones clave de la nutrición para la promoción de adaptaciones favorables al entrenamiento físico.

\section{Diferencias en las necesidades nutricionales entre atletas infantiles $y$ atletas adultos}

Aunque se dispone de datos limitados, parecería que las características fisiológicas y metabólicas principales del ejercicio en niños, que diferencian sus demandas nutricionales de las de los adultos, son las siguientes:

(1) El incremento de los lípidos en relación con la oxidación de los hidratos de carbono durante el ejercicio submáximo de intensidad relativa dada, que reduce la dependencia del glucógeno como combustible muscular y puede, en consecuencia, reducir la importancia de los hidratos de carbono alimentarios como combustible muscular $[35,44]$.

(2) La reducción de la eficiencia mecánica durante actividades que soportan peso, como marchar y correr, que resulta en un mayor gasto energético por masa corporal unitaria y limita el valor de tablas de gasto de energía para diferentes actividades derivadas de datos experimentales de adultos para uso en niños $[22,56]$.

(3) La reducción de la transpiración y la capacidad para disipar calor y regular la temperatura corporal profunda, que incrementa la necesidad de ingerir líquidos $[22,57]$.

Estas diferencias entre niños y adultos disminuyen durante la adolescencia, que se acompaña de incrementos del tamaño corporal y maduración puberal $[2,23]$. Hasta la fecha, la investigación ha llegado a la conclusión de que los niños prepuberales poseen características metabólicas y fisiológicas para rendir efectivamente en el ejercicio prolongado (aerobio) y las actividades que implican tandas cortas $(<10 \mathrm{~s})$ de actividad intensa, que son impulsadas por la energía derivada de la descomposición de la ATP y la creatina fosfato, como los saltos, los lanzamientos y las carreras cortas a toda velocidad. No obstante, parecería que los niños prepuberales no están bien dotados fisiológicamente para realizar actividades que estén sustentadas por la glucolisis anaerobia [22,23]. En el curso de la adolescencia, particularmente en muchachos, se produce un incremento de la capacidad para la glucolisis anaerobia, que puede ser mediada a través de un desarrollo del control enzimático de esta vía respiratoria y/o un incremento de la capacidad para el almacenamiento de glucógeno [22, 23]. Durante el ejercicio aerobio, con el incremento de la intensidad del ejercicio, los niños, igual que los adultos, metabolizan relativamente más depósitos endógenos de hidratos de carbono (es decir, glucógeno en el músculo y glucosa en la sangre) y, en proporción, menos grasa $[22,44]$. No obstante, es interesante destacar que los niños parecen oxidar relativamente más hidratos de carbono exógenos ingeridos durante el ejercicio que los adultos [34, 35]. Esto puede deberse a que la capacidad para catabolizar glucógeno endógeno para uso como sustrato energético por el músculo en funcionamiento es menor en niños que en adultos.

Los niños con un nivel elevado de actividad física, sobre todo los sometidos a un entrenamiento físico extenuante para practicar un deporte competitivo, presentan necesidades de energía y nutrientes alimentario específicos que sobrepasan a las de niños sedentarios o con actividad normal. No obstante, actualmente, debido a las limitadas pruebas de investigación disponibles, no es evidente de qué manera las necesidades nutricionales de los niños atletas difieren de las de sus contrapartes con actividad normal. En adultos se ha constatado que estrategias alimentarias específicas, que consisten en el consumo de hidratos de carbono con o sin proteínas, antes, durante y 
después del ejercicio, fomentan el rendimiento físico y la recuperación después del ejercicio $[21,58]$. Las estrategias de alimentación con hidratos de carbono aumentan las reservas de glucógeno antes y después del ejercicio, lo que contribuye a mantener una concentración adecuada de glucosa en la sangre y a limitar el catabolismo del glucógeno y las proteínas durante el ejercicio $[20,28]$. En el momento actual, el grado en el cual este tipo de procedimiento alimentario intensifica el rendimiento y la recuperación, después del ejercicio de los niños, es en gran medida especulativo. Debe de adoptarse una actitud precavida sobre el empleo de aportes complementarios de alimentos tanto en niños [59] como en adultos [8].

\section{Balance de líquidos y termorregulación}

El balance hidroelectrolítico es crucial para el mantenimiento de la homeostasis y el rendimiento físico. La actividad muscular es en gran medida ineficiente, dado que más del 75\% de la energía que procede de los combustibles metabólicos se transforma en calor, en lugar de en energía mecánica. Esto se confirma especialmente en niños, que presentan una eficiencia mecánica menor que los adultos durante las marchas y las carreras $[22,56]$. El almacenamiento excesivo de calor dentro del organismo causa hipertermia y golpe de calor. Esto es así a pesar de la puesta en práctica de respuestas compensatorias a una elevación de la temperatura corporal profunda, que incluye vasodilatación, que incrementa la convección del calor a la superficie cutánea, y el sudor, que incrementa la pérdida de calor a través de la evaporación y enfría la piel.

La incapacidad para reemplazar los líquidos y los electrolitos (especialmente sodio) perdidos a través de la transpiración da lugar a deshidratación y desequilibrios electrolíticos (por ejemplo, hiponatremia) [22, 57]. Por otra parte, los niños presentan un mayor riesgo de golpe de calor que los adultos, debido a un mayor cociente entre el área de la superficie y el volumen, un menor gasto cardíaco, una menor eficiencia mecánica y respuestas compensatorias subdesarrolladas a la hipertermia $[22,57]$. El riesgo de golpe de calor y deshidratación es máximo cuando la temperatura ambiental supera los $37^{\circ} \mathrm{C}$ y el nivel de humedad es demasiado elevado para permitir una pérdida de calor por evaporación efectiva. Entre los signos de termopatía destacan la excreción de escasas cantidades de orina oscura, del color de las bebidas de cola, letargia, náusea, cefalea y confusión.

El reemplazo de los líquidos perdidos es necesario para mantener el gasto cardíaco, la presión arterial y la perfusión tisular. Esta última contribuye al suministro de nu- trientes y oxígeno y a la eliminación de desechos metabólicos. Dado que la sed es en general un indicador deficiente de las necesidades de líquidos, tanto en adultos como en niños, es importante alentar a la ingestión de líquidos antes, durante y después del ejercicio, para prevenir la deshidratación. La adición de pequeñas cantidades de cloruro sódico al agua sensibiliza el mecanismo de la sed a través del mantenimiento de la osmolalidad plasmática y reduce el efecto diurético del consumo exclusivo de agua $[8,22,57]$. Para las tandas de ejercicios que sobrepasan períodos de 1 hora, es probable que, tal como se ha observado en adultos, la adición de hidratos de carbono evite la hipoglucemia y pueda mejorar tanto el rendimiento como la recuperación después del esfuerzo $[8,22$, 57].

\section{Recomendaciones prácticas}

El objetivo principal de este artículo es el de justificar las necesidades nutricionales de niños atletas basándose en las demandas fisiológicas y metabólicas de supervivencia, crecimiento y actividad física. No obstante, existen cuestiones prácticas obvias que deben considerarse en el momento de alentar al consumo de una alimentación adecuada en dichos niños. Estas cuestiones son del interés tanto de los padres como de los preparadores y los propios niños, para quienes las pruebas científicas tienen que traducirse en una práctica conductual. Las medidas prácticas para conseguir una dieta equilibrada han sido abordadas en detalle previamente [22, 24]. También puede obtenerse asesoramiento informativo en sitios web como el Instituto de Ciencia Deportiva Gatorade (www.gssiweb.com) y el Centro de Política Nutricional del Departamento de Agricultura de Estados Unidos (www.usda.gov/cnpp). Este último informa de la 'Guía Piramidal Alimentaria' ilustrada (www.mypyramid.gov), que se ha diseñado para proporcionar medios fácilmente comprensibles para que el público acceda a una alimentación nutritiva y bien equilibrada. Esta guía clasifica los alimentos por su fuente y constituyentes nutricionales principales (por ejemplo, lácteos, carne/pescado, verduras, frutas y cereales) y recomienda cantidades que deben ser consumidas por adultos y niños basándose en tamaños de raciones que proporcionan una ingesta apropiada de todos los nutrientes esenciales. Para adolescentes mayores (16 a 18 años) son aplicables y apreciables las recomendaciones alimentarias que se imparten en la Declaración de Posición Conjunta sobre Nutrición y Rendimiento Atlético de ACSM, ADA y DC [8]. 
Tal como se ha explicado preliminarmente, la energía y las proteínas alimentarias son especialmente pertinentes para el mantenimiento del estado de salud y el rendimiento físico de atletas en fase de crecimiento. La cantidad de energía 'extra' exigida para el crecimiento varía de acuerdo al tamaño corporal y la madurez y se prescribe apropiadamente en relación con la masa corporal en niños sin obesidad. Las demandas de energía y proteínas son máximas durante los brotes de crecimiento puberal y son mayores en chicos que en chicas debido a la mayor tasa de incremento del tamaño y peso corporal en los primeros [ $[1$, 24]. Durante esta fase de desarrollo, que acontece normalmente en torno a los 8 a 10 años de edad, las niñas y los niños con actividad normal suelen necesitar de 65 a $75 \mathrm{kcal} / \mathrm{kg} /$ día, junto a una ingesta de proteínas de $1,1 \mathrm{a}$ 1,2 g/día. A los 12 a 13 años de edad, las necesidades de energía y proteínas declinan hasta 50 a $60 \mathrm{kcal} / \mathrm{kg} /$ día y 1,0 a $1,1 \mathrm{~g} /$ día, respectivamente, y a los 16 o 17 años de edad, hasta 40 a $50 \mathrm{kcal} / \mathrm{kg} /$ día y 0,9 a 1,0 g/día, respectivamente [22,24]. Las demandas de energía y proteínas más elevadas se aplican en general a los varones debido a su mayor tamaño. El coste energético del entrenamiento físico debe tenerse en cuenta y añadirse a estas necesidades.

En niños sanos, las elevadas demandas de energía, asociadas al entrenamiento físico, se logran habitualmente a través de un incremento natural del apetito, y es importante que esta energía derive de una alimentación nutritiva y bien equilibrada. La adhesión a la Guía Piramidal Alimentaria suministra energía en proporciones de macronutrientes aproximadas del $55 \mathrm{al} 60 \%$ de hidratos de carbono, 12 al $15 \%$ de proteínas y 25 al $30 \%$ de grasa. Actualmente, los datos son escasos para calificar los efectos del entrenamiento físico sobre las necesidades proteínicas de atletas en fase de crecimiento. No obstante, es factible que, tal como se demuestra en atletas maduros [21], los atletas infantiles requieran del 50 al $80 \%$ más de proteínas que sus contrapartidas con actividad normal, para prevenir el balance nitrogenado negativo. No obstante, es probable que si se asegura el mantenimiento del balance energético y las proporciones de macronutrientes alimentarios mencionadas anteriormente, se cubrirán adecuadamente las necesidades de proteínas e hidratos de carbono adicionales de los atletas en crecimiento. Los hidratos de carbono son 'ahorradores de proteínas', dado que limitan el uso de las proteínas como sustrato de la síntesis de ATP y la gluconeogénesis $[8,24]$. Es posible un riesgo de deficiencia proteínica en niños que siguen una dieta vegetariana, especialmente si se excluyen el pescado y los productos lácteos que, lo mismo que la carne, contienen todos los aminoácidos esenciales. En atletas infantiles vegetarianos, una selección prudente de alimentos proteí-

Las demandas nutricionales del ejercicio nicos mixtos (por ejemplo, legumbres, cereales y frutos secos) es esencial para garantizar una ingestión equilibrada y adecuada de todos los aminoácidos esenciales.

El crecimiento y el ejercicio regular también exigen el consumo de alimentos que proporcionen cantidades suficientes de micronutrientes para controlar las vías metabólicas esenciales que participan en la síntesis de ATP y tejido nuevo. En el curso del crecimiento, se precisan hierro y calcio en cantidades elevadas para la síntesis de hemoglobina y mineral óseo, respectivamente. Cabe destacar por su importancia que en estudios nutricionales, realizados en niños con actividad normal en EE.UU. [24] y el Reino Unido [60], se han demostrado deficiencias de esos minerales concretos, así como de las vitaminas C, E y $\mathrm{B}_{6}$. Aunque estas carencias son atribuibles en gran medida a deficiencias alimentarias, un incremento relacionado con el crecimiento de la demanda de estos micronutrientes es también un factor contributivo. Los niños físicamente activos deben ser alentados a ingerir líquidos con regularidad ( $100 \mathrm{a} 150 \mathrm{ml} / \mathrm{h}$ ) durante todo el día y, en función del tamaño corporal, consumir una cantidad extra de 200 a $400 \mathrm{ml}$ de agua en el curso de las 2 horas precedentes al entrenamiento, 150 a $200 \mathrm{ml}$ cada 15 a $20 \mathrm{mi}-$ nutos durante el ejercicio y 400 a $600 \mathrm{ml}$ inmediatamente después del entrenamiento [57]. El mantenimiento del balance hídrico y el estado de hidratación en los niños puede intensificarse mediante el suministro de bebidas muy apetecibles (por ejemplo, aromatizadas).

\section{Conclusiones}

Los objetivos principales de la infancia son el crecimiento y la maduración óptimos, que exigen un balance energético y nitrogenado positivo. En consecuencia, los niños que realizan deportes extenuantes tienen que consumir una alimentación que satisfaga adecuadamente las demandas nutricionales, tanto de su entrenamiento físico como de su crecimiento normal. Las necesidades alimentarias de los niños físicamente activos varían a medida que aumenta su tamaño corporal y la progresión de su pubertad. El estado de maduración influye sobre la capacidad de rendimiento físico y las necesidades alimentarias a través de sus efectos sobre las respuestas metabólicas y fisiológicas al ejercicio. Los niños prepuberales están bien dotados físicamente para afrontar tandas prolongadas de actividades aerobias; sin embargo, su rendimiento anaerobio es limitado. Igual que los adultos físicamente activos, los niños activos tienen mayores demandas alimentarias de energía, hidratos de carbono, proteínas y agua que sus contrapartidas con actividad normal o sedentarias.

Ann Nestlé [Esp] 2006;64:63-76 


\section{Bibliografía}

1 Malina RM: Physical growth and biological maturation of young athletes. Exerc Sports Sci Rev 1994;22: 389-433.

2 Naughton G, Farpur-Lambert NJ, Carlson J, Bradney M, Van Praagh E: Physiological issues surrounding the performance of adolescent athletes. Sports Med 2000;30:309-325.

$\checkmark 3$ Roemmich JN, Richmond EJ, Rogel AD: Consequences of sport training during puberty. J Endocrinol Invest 2001;24:708-715.

4 Zanker CL Cooke CB: Energy balance, endocrine function and bone health. Med Sci Sports Exerc 2004;36:1372-1381.

5 Soyka LA, Grinspoon S, Levitsky LL, Herzog DB, Klibanski A: The effects of anorexia nervosa on bone metabolism in female adolescents. J Clin Endocrinol Metab 1999;84:4489-4496.

6 Warren MP: The effects of exercise on pubertal progression and reproductive function in girls. J Clin Endocrinol Metab 1980;51:1150-1157.

7 Rogol AD, Clark PA, Roemmich JN: Growth and pubertal development in children and adolescents: effects of diet and physical activity. Am J Clin Nutr 2000;72(suppl):521S-528S.

8 Manore MM, Barr SI, Butterfield GE: Nutrition and Athletic Performance. Joint Position Statement of ACSM/ADA/DC. Med Sci Sports Exerc 2000;32: 2130-2145.

9 Medical Research Council Ethics Guide. Medical Research Involving Children. London, Medical Research Council, 2004.

10 McIntosh N, Bates P, Brykczynska G, Dunstan G, Goldman A, Harvey D, Larcher V, McCrae D, McKinnon A, Patton M, Saunders J, Shelley P. Guidelines for the ethical conduct of medical research involving children. Royal College of Paediatrics and Child Health: Ethics Advisory Committee. Arch Dis Child 2000;82:177-182.

11 International Ethical Guidelines for Biomedical Research Involving Human Subjects. Geneva, Council for International Organizations of Medical Sciences, 2002.

$>12$ De Feo P, Di Loreto C, Lucidi P, Murdolo G, Parlanti N, De Cicco A, Piccioni F, Santeusanio F: Metabolic response to exercise. J Endocrinol Invest 2003 26:851-864.

13 Hagerman FC: Energy metabolism and fuel utilization. Med Sci Sports Exerc1992;24:S309-S314.

14 Ainsworth BE, Haskell WL, Whitt MC, Irwin ML, Schwartz, AM, Strath SJ, O'Brien WL, Bassett DR Jr, Schmitz KH, Emplaincourt PO, Jacobs DR Jr, Leon AS: Compendium of physical activities: an update of activity codes and MET intensities. Med Sci Sports Exerc 2000;32:S498-S504.

15 Hendricks KM, Duggan C, Walker WA: Manual of Pediatric Nutrition, ed 3. Hamilton, Decker, 2000 p 142.

$>16$ Brooks GA: Mammalian fuel utilization during sustained exercise. Comp Biochem Physiol Biochem Mol Biol 1998;120:89-107.

17 Coyle EF: Substrate utilization during exercise in active people. Am J Clin Nutr 1995;61(suppl):S968S979.

18 Tarnopolsky LJ, MacDougall JD, Atkinson SA, Tarnopolsky MA, Sutton JR: Gender differences in substrate for endurance exercise. J Appl Physiol 1990;68 302-308.

19 Horton TJ, Pagliassotti MJ, Hobbs K, O’Hill J: Fuel metabolism in men and women during and after long-duration exercise. J Appl Physiol 1998;85: 1823-1832.

20 Spriet LL, Peters SJ: Influence of diet on the metabolic responses to exercise. Proc Nutr Soc 1998;57: 25-33.
21 Kreider RB, Miriel V, Bertun E: Amino acid supplementation and exercise performance. Analysis of the proposed ergogenic value. Sports Med 1993;16:190206.

22 Bar-Or O: Nutritional considerations for the child athlete. Can J Appl Physiol 2001;26(suppl):S186S191.

23 Boisseau N, Delamarche P: Metabolic and hormonal responses to exercise in children and adolescents: Sports Med 2000;30:405-422.

24 Steen SN: Nutrition for the school age athlete; in Berning JR, Steen SN (eds): Nutrition for Sport and Exercise. Gaithersburg, Aspen, 1998, pp 217-245.

25 Green HJ: Mechanisms of muscle fatigue in intense exercise. J Sports Sci 1997;15:247-256.

26 Romijn JA, Coyle EF, Sidossis LS, Gastaldelli A, Hoeowitz JF, Endert E, Wolfe RR: Regulation of endogenous fat and carbohydrate metabolism in relation to exercise intensity and duration. Am J Physiol 1993;265:E380-E391.

27 Brooks GA, Mercier J: Balance of carbohydrate and lipid utilization during exercise: the 'crossover' concept. J Appl Physiol 1994;76:2253-2261.

28 Wagenmakers AJM, Beckers EJ, Brouns F, Kuipers H, Soeters PB, Van Der Vusse GJ, Saris WHM: Carbohydrate supplementation, glycogen depletion and amino acid metabolism during exercise. Am J Physiol 1991;260:E883-E890.

29 Kasperek GJ, Snider RD: Effect of exercise intensity and starvation on activation of branched-chain keto-acid dehydrogenase by exercise. Am J Physiol 1987;252:E33-E37.

30 Zanker CL, Swaine IL, Castell L, Newsholme EA: Responses of plasma glutamine, free tryptophan and branched-chain amino acids to prolonged exercise after a regime designed to reduce muscle glycogen. Eur J Appl Phys 1997;75:543-548.

31 Galbo H: Exercise physiology: humoral function. Sport Sci Rev 1992;1:65-93.

32 Viru A: Stability and variability in hormonal responses to prolonged exercise. Int J Sports Med 1992;13:230-234

33 Martinez LR, Haymes EM: Substrate utilization during treadmill running in prepubertal girls and women. Med Sci Sports Exerc 1992;24:975-983.

34 Riddell MC, Bar-Or O, Wilk B, Parolin ML, Heigenhauser GJ: Substrate utilization during exercise with glucose and glucose plus fructose ingestion in boys ages 10-14 yr. J Appl Physiol 2001;90:903-911.

35 Timmons BW, Bar-Or O, Riddell MC: Oxidation rate of exogenous carbohydrate during exercise is higher in boys than in men. J Appl Physiol 2002;94: 278-284.

36 Berg A, Keul J: Biochemical changes during exercise in children; in Malina RM (ed): Young Athletes: A Biological, Psychological and Educational Perspective. Champaign, Human Kinetics, 1988, pp 61-67.

37 Astrand PO, Rodahl K: Textbook of Work Physiology: Physiological Bases of Exercise. New York, McGraw-Hill, 1986, pp 295-353.

38 Biddle S, Sallis J, Cavill N: Young and Active? Young People and Health-Enhancing Physical Activity: Evidence and Implications. London, Health Education Authority, 1998.

39 Zanker CL, Gannon L, Cooke CB, Gee KL, Oldroyd B, Truscott JG: Differences in bone density, body composition, physical activity and diet between child gymnasts and untrained children aged 7-8 years. J Bone Miner Res 2003;18:1043-1050.

40 Caine D, Lewis R, O'Connor P, Howe W, Bass S: Does gymnastics training inhibit growth of females? Clin J Sport Med 2001;11:260-270.

41 Armstrong N, Welsman JR: Developmental aspects of aerobic fitness in children and adolescents; in
Holloszy JO (ed): Exercise and Sports Science Reviews. Baltimore, Williams \& Wilkins, 1994, pp 435 476

42 Saltin B: Oxygen transport by the circulatory system during exercise in man; in Keul J (ed): Limiting Factors of Physiological Performance. Stuttgart, Thieme, 1973, pp 235-252.

43 Pate RR, Kriska A: Physiological basis of the sex difference in cardiorespiratory endurance. Sports Med 1984;1:87-90.

44 Foricher JM, Ville N, Gratas-Delamarche A, Delamarche P: Effects of submaximal intensity cycle ergometry for one hour on substrate utilization in trained prepubertal boys versus trained adults. Sports Med Phys Fitness 2003;43:36-43.

45 Golden K, Waterlow JC, Picou D: The relationship between dietary intake, weight change, nitrogen balance and protein turnover in man. Am J Clin Nutr 1977;30:1345-1348.

46 Matkovic V, Ilich J, Skugor M, Badenhop NE, Goel P, Clairmont A, Klisovic D, Nahhos RW, Landoll JD Leptin is inversely related to age at menarche in human females. J Clin Endocrinol Metab 1997;82: 3239-3245.

47 Welt CK, Chan JL, Bullen J, Murphy R, Smith P DePaoli AM, Karalis A, Mantzoros CS: Recombinant human leptin in women with hypothalamic amenorrhea. N Engl J Med 2004;351:987-997.

48 Bonjour J, Theintz G, Buchs B, Slosman D, Rizzoli R Critical years and stages of puberty for spinal and femoral bone mass accumulation during adolescence. J Clin Endocrinol Metab 1991;73:555-563.

49 Weaver CM: Adolescence: the period of dramatic bone growth. Endocrine 2002;17:43-48.

50 Baxter-Jones ADG, Helms PJ: Effects of training at a young age: review of the training of young athletes study. Pediatr Exerc Sci 1996:8:310-327.

51 Wilson GT, Eldridge KL: Pathology and development of eating disorders: implications for athletes; in Brownell KD, Rodin J, Wilmore JH (eds): Eating, Body Weight and Performance in Athletes. Disorders of Modern Society. Philadelphia, Lea \& Febiger, 1992, pp 115-127.

52 Sundgot-Borgen J: Risk and trigger factors for the development of eating disorders in female elite athletes. Med Sci Sports Exerc 1994;26:414-419.

53 Viru A: Post-exercise recovery period: carbohydrate and protein metabolism. Scand J Med Sci Sports 1996;6:2-14

54 Kuipers H, Keizer HA: Overtraining in elite athletes: a review and directions for the future. Sports Med 1988;6:79-92.

55 Fry RW, Morton AR, Keast D: Overtraining in athletes: an update. Sports Med 1991;12:32-65.

56 Frost G, Dowling J, Dyson K, Bar-Or O: Cocontraction in three age groups of children during treadmil locomotion. J Electromyogr Kinesiol 1997;7:179186.

57 Rivera-Brown AM, Gutierrez R, Gutierrez JC, Frontera WR, Bar-Or O: Drink composition, voluntary drinking and fluid balance in exercising, trained, heat-acclimatized boys. J Appl Physiol 1999;86:7884.

58 Zawadski KM, Yaspelkis BB, Ivy JL: Carbohydrateprotein complex increases the rate of muscle glycogen storage after exercise. J Appl Physiol 1992;72: 1854-1859.

59 Johnson WA, Landry GL: Nutritional supplements: fact vs. fiction. Adolesc Med 1998;9:501-513.

60 Gregory J, Lowe S, Bates CJ, Prentice A, Jackson LV, Smithers G, Wenlock R, Farron M: National Diet and Nutrition Survey: Young People Aged 4 to 18 Years. Vol 1: Report of the Diet and Nutrition Survey London, TSO, 2000. 